$1-1996$

\title{
Basics of research (Part 5): Ethics and human rights
}

Eric Davis

Strong Memorial Hospital

Edward A. Panacek

University of California, Davis

Cheryl Thompson

University of Nebraska Medical Center, cbthompson@unmc.edu

Tell us how you used this information in this short survey.

Follow this and additional works at: https://digitalcommons.unmc.edu/con_articles

Part of the Nursing Commons

\section{Recommended Citation}

Davis, Eric; Panacek, Edward A.; and Thompson, Cheryl, "Basics of research (Part 5): Ethics and human rights" (1996). Journal Articles: College of Nursing. 18.

https://digitalcommons.unmc.edu/con_articles/18

This Article is brought to you for free and open access by the College of Nursing at DigitalCommons@UNMC. It has been accepted for inclusion in Journal Articles: College of Nursing by an authorized administrator of DigitalCommons@UNMC.For more information, please contact digitalcommons@unmc.edu. 


\title{
Basics of Research (Part 5): Ethics and Human Rights
}

\author{
Eric A. Davis, MD, ${ }^{1}$ Edward A. Panacek, MD,2 Cheryl Bagley Thompson, PhD, RN, Cs 3
}

1. STAT Medevac, Center for Emergency Medicine, Pittsburgh, $\mathrm{Pa}$.

2. Division of Emergency Medicine and Clinical Toxicology, University of California, Davis, Medical Center, Sacramento, Calif.

3. University of Utah College of Nursing, Salt Lake City, Utah

Key Words: ethics, informed consent, research

\section{Introduction}

The concept of ethical treatment of human subjects has a long and constantly evolving history. Its roots are found in ancient Anglo common law, but it was not until the 20th century, as the abuses inflicted on research participants came to light, that it attained the importance it holds today. Currently, all studics involving humans are subject to extensive regulations, requiring the project to be approved by an institutional review board (IRB) - a body charged with protecting the patients rights while fostering valid and methodologically sound research. ${ }^{1}$ Informed consent-the prime component of the process by which the patient is provided information to make a determination as to whether to participate in any experimental therapy or process-must be addressed for each project. The purpose of this paper is to address broadly issues regarding ethics and prehospital research, focusing on informed consent.

\section{Historical Perspective}

The history of informed consent with respect to treatment was first commented on by Plato, who believed that the physician should expressly not discuss care with the patient. He stated, "You are not healing the sick man, but you are educating him, and he does not want to be made a doctor but to get well." Ancient law expanded this concept, but provided minimal human rights protection and limited the authority of the physician to treat only those who requested it. However, on this request, which was considered to be implied by any person presenting to the physician, the patient could be sub- jected to all treatment the doctor deemed necessary without the right to withdraw from or even inquire about the therapy being rendered.

Modern ethical issues have their roots in Anglo-American common law, which "forbid harmful or offensive nonconsensual touching, however benign in motive or physical effect without their consent." Here, the first definition of assault-a threat of approach, battery and touching intentionally-came into use. The only exception was in emergency situations. The content of consent was not defined, it was interpreted very broadly, and could take the form of actions, words, or be implied from the circumstances. The first case law occurred in 1914, when Supreme Court Justice Cardoso ruled that "Every human being of adult years and sound mind is entitled to determine what is to be done with his own body."3 Despite this, cthical issues and informed consent remained poorly defined and were left to the judgment of the researcher. A series of horrific situations changed all that, and brought the issues into focus, eventually leading to our current regulations.

The first event was the revelation of the Nazi atrocities in World War II in the name of research uncovered during the Nuremberg trials. This led to the development of the Nuremberg Code in 1949, which became the cornerstone of current research ethics. ${ }^{4}$ Five main concepts are at the heart of the code:

1. Voluntary consent by the research subject

2. Explanation of all potential hazards

3. The research produces fruitful re 


\section{Table 1}

\section{Criteria for Institutional \\ Review Board Approval}

1. Hisks to the patient are minimized.

2. Risks are reasonable in relation of benefits, including the benefit of knowledge gained.

3. Equitable subject selection.

4. informed consent sought and documented.

5. Privacy of the subjects is maintained strictly.

6. Ongoing monitoring of data to ensure safety.

7. Safeguards are included to protect the rights and welfare of vulnerable subjects.

sults for the good of society

4. Valid animal-model based studies

5. Each subject can stop the experiment at any time.

This code spawned a major development of case law, and while the vast majority concerned medical practice, the rulings and principles are generally thought to hold true for research consent. To summarize, consent deals with the ethical principle of respect for persons. The consent process, in theory, is supposed to ensure that the researcher will respect the subject to be self-determining and able to make independent free choices. Unless the person allows it, the investigator is not allowed to violate this tenet and touch or enter their private space. The traditional legal view of violation of this principle and failure to obtain adequate consent is battery. Whether or not harm results is irrelevant. More recent case law treats failure to obtain consent as negligence. Negligence expands on the previous legal view and brings in the concept of standard therapy and whether the treatment is deviating from standard therapy (as in all experimental research). If so, then failure to obtain proper informed consent may be treated as a negligent action. The traditional components of a negligent action may then apply (duty, breach, damage and casualty). In both doctrines, the consent is held to be invalid if any information thought to be necessary or important in the subject's decision-making process is withheld. Additionally, it is incumbent on the researcher to prove the subject has grasped the material prior to consenting.

A brief mention also should be made concerning two further ethical codesthe Declaration of Helsinki and the Belmont Report.5,6 The thrust of the former is to ensure sound, ethical and valuable research. The responsibility for subject welfare is placed squarely on the shoulders of the researcher. It also recommended strict accuracy of data/ results in the publication, with a prohibition against unethical research being published. The Belmont Report is a more detailed analysis of basic ethical principles for both medical practice and research. The first deals with respect for persons, recognizing an individual's right to self-determination and protecting those with diminished autonomy through the process of informed consent. The next principle, beneficence, strives to maximize benefit while minimizing possible risks. This is to be guaranteed through careful, stringent, and systematic assessment of risks and benefits for each study. Justice, or the principle of fairness with respect to the distribution of benefit, as well as burden of research, is determined through fair selection processes that pay close attention to possible bias concerning sex, race, societal and cultural issues.

While these developments were a positive step in aiding ethical research practices, the onus to ensure they were applied properly was still left entirely to the researcher. A series of incidents would soon change this and bring swift and vociferous protests from both public and scientific communities. A succession of highly unethical studies was publicized, the most famous of which was the Tuskeegee Syphilis study, in which a group of African-American males infected with the disease continued to be observed for effects to study the natural course of the disease, even after treatment (penicillin) became available, 2,7 Other studies injected elderly patients with cancer cells without their knowledge, and intentionally exposed mentally impaired patients to Hepatitis $\mathrm{A}^{2}$ The concept of the benevolent researcher always striving to protect the study subjects was forever shattered, and led to the current stringent regulations overseeing human research.

\section{Institutional Review Boards}

In 1966, the U.S. Public Health Service mandated that all federally funded research involving human subjects requires review by a "committee of institutional associates." 1,7 The mission of this committee was to ensure that the autonomy of the subject was respected, that a well-defined benefit would result from this research, and that vulnerable subjects (e.g., children or prisoners) would be protected. This initiated the widespread adoption of the institutional review board (IRB). The IRB concept was further developed in the early 1970 s when the Public Health Service required peer review for any research supported by Department of Health Education and Welfare grants. Subsequent to this, a series of congressional hearings was held in 1974 and established the requirement for IRBs at all institutions receiving any federal grants, and established the National Commission for the Protection of Human Subjects of Biomedical and Behavioral Research. The code of federal regulations was revised in 1983 and was adopted by the Department of Health and Human Services (DHHS) Office for Protection for Research Risks (OPRR). ${ }^{1}$ This set the criteria necessary for IRB approval (Table 1). These are general guidelines, and each IRB has specific instructions as to the form and content of each research proposal. This is available from each individual IRB office. The directive also lists specific steps on how to carry out the process of reviewing research to ensure that it meets the standards. It is important to note that the IRB has been given the right to preview all qualifying research proposals to ensure these criteria are met and that a valid study will result.

Institutional review boards vary in their procedures. Most require that the investigator submit a written proposal 
and standardized request forms for review by the committee. Although all research should be submitted through the IRB process, some projects will be classified by the IRB as minimal risk and, as a result, receive an expedited review. Types of research that may be classified as minimal risk are chart reviews and anonymous surveys. Although the investigator may be confident that the study is minimal risk and, thus, does not require IRB approval, all proposals should be submitted for review and patient confidentiality. This places the responsibility for the review on the IRB, minimizing investigator responsibility should the study be inappropriately classified as exempt from review.

The process for an expedited review varies across institutions but often involves review by a single staff member rather than by the entire committee. Because an expedited review does not involve the entire committee, the time needed to complete the review is shortened. Investigators should determine the time needed to obtain the appropriate IRB approval early in the planning stages of the project.

The next step in dealing with the IRB is to decide whether the proposed study meets the criteria for full committee review. The definition of this states the IRB has jurisdiction in "all research involving human subjects conducted, supported or otherwise subject to regulation by any federal department or agency." 1 Also, all human-subject research performed in an institution with written DHSS-approved assurances applies. Put simply, any study involvement that performs or alters any human intervention must be IRB approved. There are also other considerations that each individual IRB may want to review for proper confidentiality, institutional or personal liability or other potential problems. These regulations concerning each particular IRB can be obtained from the IRB office.

Suggestions for dos and don'ts when dealing with the IRB are listed in Table 2 . The interaction between the researcher and the IRB is an involved process. Attempts to properly interact with the IRB members for both education and explanation purposes can yield dramatic results and greatly improve the

Table 2

\title{
Dos and Don'ts for Interacting with the Institutional Review Board
}

\author{
Do: \\ Design a good project \\ Know intricate details of the project \\ Show concern for protecting your patients \\ Use lay language in consent forms and presentation \\ Allow realistic time for review and modification \\ Know individual IRB members \\ Acquaint the chairperson with your protocol \\ Don't: \\ Argue with the IRB \\ Submit a proposal in an improper form \\ Use technical language \\ Disregard patient rights and protection \\ Submit a proposal at the last minute \\ Be unfamillar with any aspect of a project
}

project. However, this process takes time, and it is unrealistic to believe that each proposal will be approved on its initial submission. The investigator must understand that the IRB has a difficult job, and that board members are trying to ensure good and proper research and not trying to delay the project.

Air transport programs that are not associated with a single health-care organization or academic institution and have not received federal funding may not have an official IRB. Because of the protection offered to the researcher by the IRB, such programs may wish to associate themselves with a sponsoring hospital or develop their own procedures for review of proposed investigations.

Multi-center research presents additional challenges to obtaining IRB approval. The principal investigator and project coordinator at all data collection sites must obtain IRB approval at their respective institutions. This requirement can create difficulties when two IRBs have inconsistent requirements of the investigators. A common area of difficulty lies in the consent form. Individual institutions may require specific words to be placed in their consent forms, and these requirements may be in direct conflict with one another. If inconsistent consent forms are required, the researcher may be served best by having the subjects sign two consent forms-the one from the institution of the principal investigator and the one from the institution where data are be- ing collected on that subject. Subject consent forms may be kept at the institution where the data are collected or all may be stored by the principal investigator. In either case, the consent forms must be available for review by federal or other agencies on request.

\section{Informed Consent}

The cornerstone of each IRB proposal and the area most scrutinized is that of informed consent. Without adequate preparation and attention to this area, the research proposal will not gain the necessary approval from the IRB. Absence of appropriate informed consent also opens the researcher and the sponsoring institution to civil liability. 8

To obtain valid informed consent, four basic tenets must be met. 2,3,7,9 The first is that the subject has adequate decision-making capacity, including a proper emotional state that allows a decision to be made. The patient must be legally competent and under no coercion. Finally, all relevant information must be disclosed in an understandable manner to the subject. Several points deserve explanation. Many ethicists feel that the process of reviewing the consent with the patient is inherently flawed, especially if a physician is participating and does not allow the subject to make a free choice. There are many motives for patients to participate in research: one is to please the reviewer, a factor that can become magnified when it is the physician who reviews the con- 
Table 3

\section{Department of Health and Human Services Consent Waiver Requirements}

1. The research could not practicably be carried out without the waiver.

2. Whenever appropriate, the subject will be provided with additional, pertinent information after participation.

3. The research involves no more than minimal risk.

4. The waiver will not affect adversely the rights and welfare of the subject.

\section{Table 4}

\section{Food and Drug Administration Consent Waiver Requirements}

1. Subject is confronted by a life-threatening situation, necessitating the experimental therapy.

2. Informed consent cannot be obtained from the subject due to an inability to communicate or obtain legally effective consent from the subject.

3. Insufficient time to obtain consent from the subject's legal representative.

4. There is no reliable alternative method of approval therapy that provides an equal or greater likelihood of saving the life of the subject.

sent. However, unlike a normal business contract in which the burden of understanding is on the consumer, here the responsibility lies with the researcher. Imagine if when you purchase a car, the dealer was required to inform you of all the possible faults of the auto. This is the situation with research.

The next process is the actual administration of the consent, which is also well-defined. ${ }^{7}$ The first step is informing. This is a detailed 16 -step process that dictates the key elements of information that must be communicated to the subject. While each step is not valid for every project, the basic framework must be followed. Next is an assessment of the subject's comprehension of the presented material, and proof of understanding may be required. Interestingly, the material needs only to be understood at the time of presentation, not in the future. Following this, an assessment of the subject's autonomy must be conducted, and he or she must not be under any undue stress. The last step is the demonstration by the prospective participant of a willingness to participate, which forms the actual act of consenting. In the majority of cases, this includes the completion of a written form with a signature, although other methods may be acceptable.

There are a number of problems with the classical approach to consent, particularly in the prehospital setting. Research into the consent procedures, and how it is accepted by patients, has demonstrated that research subjects often do not understand the process, and may sign the form for such reasons as believing it necessary to receive treatment, or to please the researcher. ${ }^{8}$ When they do understand the purpose, they often prefer to have the decision made by the physician. In the prehospital setting, practical considerations limit the utility of traditional informed consent. First, the process is lengthy and time consuming. Second, it requires a competent and understanding patient and a qualified person to administer the consent (usually a physician). Obviously, these requirements would exclude the majority of patients and, therefore, most EMS research. A directive, issued in 1993 by the OPRR, mandates that all human studies approved by an IRB require prospective informed consent from the patient or patient representative. In response to the acknowledged hardship on emergency research, a meeting has been convened by the Food and Drug Administration (FDA) and the National Institutes of Health to obtain public and scientific input on the impact of consent regulations on emergency research. Until these issues are resolved, waiver regulations exist.

There are two sets of waiver regulations-one each issued by the Department of Health and Human Services (DHSS), the other by the FDA. ${ }^{2}$ The first (DHSS) permits a waiver if the IRB "finds and documents" four conditions (See Table 3). The first point is easily satisfied by the acute nature of prehospital illness, qualification of those applying the consent and the time constraints essentially preventing traditional consent. Many therapies are also time-dependent. Point two presents little problem and point four must be addressed by ensuring any preference of the patient is strictly honored, such as an advance directive.

The most difficult and complex idea involves the "condition of minimal risk." Minimal risk is defined as the risk of harm anticipated in the proposed research not being greater than those ordinarily encountered in daily life or during the performance of routine physical or psychological exams or tests. Thus, the experimental therapy should not entail more than this additional risk when compared to standard therapy. This definition has limited utility, as many of the disease processes studied in the prehospital setting, especially in air medical transport, (such as cardiac arrest, trauma and shock) inherently have poor outcome regardless of the therapy applied.

The second set of waiver regulations is provided through the FDA and governs research on unapproved drugs and devices (Table 4). Points one through three are usually easily satisfied, while point four may present problems. If any physician feels certain that one therapy is supcrior, he or she is permitted (some would say ethically bound) to use this therapy. Therefore, some have objected to the randomization process, in which a patient cannot predict which therapy he or she will receive. In such cases, there must be a real question about which treatment is superior, regardless of individual opinion. History has shown the need for objective research studies, despite anecdotal experience and opinions to be contrary. Even when there is thought to be a "clear" advantage to a particular therapy, in as many as $50 \%$ of cases this preference has proven incorrect when formally studied. ${ }^{2}$

There also has been significant discussion about the concept of deferred consent. ${ }^{3}$ This has been used in the set- 
ting of resuscitation research in which the patient is enrolled into the study without approval from the subject or family. Once the subject is stabilized and the initial experimental therapy initiated, the family is approached for traditional informed consent. At that point, the family may choose to discontinue the experimental treatment. This alternative is currently not an option as determined in the 1993 OPRR directive. This leaves only two options: informed consent or waiver.

In light of the traditional waiver requirement and the changing ideas and concerns surrounding consent, the FDA has put forth a new set of proposed guidelines titled, "Exception from Informed Consent for Emergency Research."10 This both condenses and expands on the previous two waiver regulations (Table 5). There must be a real question about current therapy, and valid scientific evidence, which may include that obtained through randomized trials, should be necessary to determine which intervention is most beneficial. No practical way can exist to obtain informed consent at the time of intervention, and prospective obtainment of consent is not feasible because the emergence of the condition being studied cannot be predicted reliably in particular individuals. The risk must be determined to be reasonable in light of what is known about the medical condition and the risks and benefits of both the current and interventional therapy. The increased provision for public disclosure and involvement makes the researchers responsible to the community in which the research is being performed and also provides input from a viewpoint that may not be considered routinely during proposal development. Consultation may be initiated by the IRB. Finally, although

Table 5

\section{Proposed Food and Drug Administration Exceptions to Consent}

1. Life-threatening situation

a. Available treatment unproven or unsatisfactory

b. Valid evidence that supports proposed study

2. Obtaining valid informed consent not feasible

a. Medical condition precludes consent

b. intervention must be administered before representative consent can be obtained

c. Prospective identification of subjects is not possible

3. Opportunity for subjects to participate is in their best interest

a. Life-threatening situation necessitates intervention

b. Risk is reasonable

4. Research could not be carried out without waiver

5. Additional protection of rights and welfare will be provided

a. Consultation from community representatives

b. Public disclosure prior to study of risks and benefits

c. Public disclosure following study

d. Development of independent data and safety monitoring board

6. International Review Board reviews and approves informed consent for situations for which it may be appropriate.

the traditional informed consent has been waived, all subjects (if not incapacitated) or their authorized representatives must be informed of their inclusion into the study, and of the details of the study. All subjects also must be informed that they may discontinue their participation at any time.

Despite the safeguards imposed by the IRB and the consent process, the prospective researcher still may have problems with ethical considerations. There are many motives for performing clinical research, which run the gamut from patient-care concerns, such as inadequate standard therapy, to those less pure, such as reimbursement and academic standing. Despite the best intentions of the researcher, studies have shown that a trial sponsored by a party with a direct stake or interest in the outcome (such as a drug company or a device manufacturer) may influence the conclusion, even if done unconscious- 1y. ${ }^{11}$ Strict attention to this possibility, such as effective blinding of subjects and researchers and use of objective data points, will help to minimize the problem. In addition, if the researcher has any financial or other outside interest in the trial, this must be mentioned in the paper. ${ }^{12}$

It is imperative that the rights and welfare of study populations be observed and guarded strictly. In the air medical transport environment, this means that all studies must be reviewed by an appropriate IRB prior to initiation of the study. Most IRBs are willing to work with the researcher to improve the proposal and to address ethical and consent issues without preventing the proposed research. The IRB processes of approval and informed consent help to ensure maintenance of the subject's rights and help to protect the researcher from claims of human rights violations.

\section{References}

1. OPRR Reports Protection of Human Subjects Code of Federal Regulations 45. CFT 46, 1974; Revised 1983.

2. Davis E, Maio R: Ethical issues in prehospital research. Prehospital and Disaster Medicine 1993;8:511-514.

3. Spivey $\mathrm{W}$, Abramson $\mathrm{N}$, Iserson $\mathrm{K}$, et al: Informed consent for biomedical research in acute care medicine. Ann Emerg Med 1991;20:1251-1265.
4. Beauchamp TL, Childress JF: The Nuremberg Code in Principles of Biomedical Ethics, New York, Oxford University Press, 1979.

5. World Medical Assembly (1975), World Medical Association Declaration of Helsinki: Recommendations Guiding Medical Doctors in Biomedical Research Involving Human Subjects. Helsinki, Finland, 1964, Tokyo, Japan.

6. The National Commission for the Protection of Human Subjects of Biomedical and Behavioral
Research: The Belmont Report: Ethical Principles and Guidelines for the Protection of Human Subjects of Research, (DHEW Publication No. (OS) 78-0012), Washington D.C., U.S. Government Printing Office, 1978.

7. Levine RJ: Ethics And Regulation of Clinical Research, 2nd ed., Urban Schawzenberg, BaltMunich 1986.

8. Lidz C, Meisel A, Osterveis M: Barriers to informed consent. Ann Intern Med 
1983;99:539-543.

9. Boisaubin E, Dressor R: Informed consent in emergency care: Illusion and reform. $A n n$ Emerg Med 1987:16:62-67.
10. Biros $\mathrm{M}$, Lewis $\mathrm{R}$, Olson $\mathrm{C}$, et al: Informed consent in emergency research. JAMA 1995;265:1283-1287.

11. Shapiro MF, Charrow RP: Scientific miscon- duct in investigational drug trials. $N$ Engl J Med 1985;312:731-736.

12. Lind SE: Fee for service research. $N$ Engl J Med 1986;314:312-315.

\section{Suggested Readings}

Kopelman L: Randomized clinical trails, consent and the therapeutic relationship. Clin Res 1983:31:1-11.

Informed consent: Study of quality of information given to participants in a clinical trial. $B M J$ 1991;303:610-613.

Meinert C: Clinical Trials, New York, Oxford University Press, 1988. research. $N$ Engl J Med 1987;317:141-145.

Lynoe N, Sandlund M, Dahlgvist G, Jacobson L:

Veatch RM: The Patient as Partner: A Theory of
Human-Experimentation Ethics, Bloomington, Ind., University Press, 1987.

Warren JW, Sobal J, Tenney JH, et al: Informed consent by proxy: An issue in research with elderly patients. N Engl J Med 1986;315:1124-1128. 